\title{
Urease activity and urea hydrolysis rate under coupling effects of moisture content, temperature, and nitrogen application rate
}

\author{
Tao Lei ${ }^{1}$, Qiongqiong $\mathrm{Gu}^{2}$, Xianghong Guo ${ }^{1}$, Juanjuan $\mathrm{Ma}^{1}$, Yong Zhang ${ }^{1}$, Xihuan Sun ${ }^{1,3^{*}}$ \\ (1. College of Water Science and Engineering, Taiyuan University of Technology, China; \\ 2. China Irrigation and Drainage Development Center, Beijing 100054, China; \\ 3. Jinzhong University, Jinzhong 030600, China)
}

\begin{abstract}
The traditional qualitative analysis of the individual factors on the kinetic parameters cannot sufficiently reveal the mechanism underlying urea hydrolysis in soil. This study aimed at revealing the coupling effects of the three factors on urease activity $\left(V_{0}\right)$, hydrolysis rate constant $\left(K_{u}\right)$, and activation energy $\left(E_{a}\right)$ and establishing the quantitative model for $K_{u}$ under the coupling condition. Laboratory culture experiments were conducted under different temperatures $(T)\left(15^{\circ} \mathrm{C}, 20^{\circ} \mathrm{C}, 25^{\circ} \mathrm{C}\right.$, and $\left.35^{\circ} \mathrm{C}\right)$, moisture contents $(\theta)(60 \%, 80 \%$, and $100 \%$ of field capacities $)$, and nitrogen application rates $(F)(247 \mathrm{mg} / \mathrm{kg}$, $309 \mathrm{mg} / \mathrm{kg}, 371 \mathrm{mg} / \mathrm{kg}$, and $433 \mathrm{mg} / \mathrm{kg}$ ). The urea content was measured daily. Results showed that the effects of moisture content, temperature, nitrogen application rate, and their interaction on $V_{0}$ and $K_{u}$ were in the descending order: $T, F, T^{*} F, \theta$, $T^{*} \theta, F^{*} \theta, T^{*} \theta^{*} F$. The effect of single factor and two-factor coupling on $V_{0}$ was extremely significant $(p<0.01)$, whereas the effect of the three-factor coupling on $V_{0}$ was negligible. The effects of three factors and their interaction on $K_{u}$ were extremely significant $(p<0.01)$. The effects of moisture content, nitrogen application rate, and their interaction on $E_{a}$ were in the descending order: $F, \theta, F^{*} \theta$. The effects of two factors and their interaction on $E_{a}$ were not significant. The mean absolute percentage error (MAPE) values of the established $K_{u-1}(\theta, T, F)$ and $K_{u-2}(\theta, T, F)$ models were $3.14 \%$ and $4.60 \%$, respectively. The MAPE of the traditional Arrhenius model $K_{u-3}(T)$ was $6.75 \%$. The accuracy of the proposed three-factor interaction model was superior to that of the traditional single factor model. The results supplemented the mechanism of urea hydrolysis and improved the prediction accuracy of $K_{u}$.
\end{abstract}

Keywords: urease activity, hydrolysis rate constant, Arrhenius model, activation energy, coupling effect DOI: $10.25165 /$ j.ijabe.20181102.3784

Citation: Lei T, Gu Q Q, Guo X H, Ma J J, Zhang Y, Sun X H. Urease activity and urea hydrolysis rate under coupling effects of moisture content, temperature, and nitrogen application rate. Int J Agric \& Biol Eng, 2018; 11(2): 132-138.

\section{Introduction}

Food security is a major agricultural topic and has been closely monitored by the Chinese government. In recent years, the food supply maintains a growing trend but still takes the old way of "high amount of fertilizer but low yield". Excessive use of chemical fertilizers exacerbates the environmental pollution ${ }^{[1-3]}$. This phenomenon has aroused great concern from the Chinese government, and the Ministry of Agriculture issued an official document for pollution control in $2015^{[4]}$. The document indicates that reducing the application rate of urea and improving the usage efficiency are effective to solve environmental pollution and promote grain production $^{[4]}$. In China, urea is most widely used in agriculture, accounting for more than $75 \%$ of the total nitrogen use. However, the use efficiency of urea is only approximately $32 \%{ }^{[5]}$. Most nitrogen amounts in fertilizers in soil are removed via

Received date: 2017-09-06 Accepted date: 2017-11-16

Biographies: Tao Lei, PhD, research interest: soil nitrogen cycle, Email: lcsyt@126.com; Qiongqiong Gu, Master, Engineer, research interest: soil nitrogen cycle, 170783854@qq.com; Xianghong Guo, PhD, Assistant Professor, research interest: soil nitrogen cycle, Email: 165305052@qq.com; Juanjuan Ma, PhD, Professor, research interest: soil nitrogen cycle, Email: 942547847@ qq.com; Yong Zhang, PhD, research interest: soil nitrogen cycle, Email: zy9506@163.com.

*Corresponding author: Xihuan Sun, PhD, Professor, research interest: soil nitrogen cycle. College of Water Science and Engineering, Taiyuan University of Technology, Taiyuan, Shanxi 030024, China. Tel/Fax: +86-0351-6111079. Email:suntyut@126.com. ammonia volatilization. Therefore, urea hydrolysis mechanism should be investigated to improve urea utilization efficiency, reduce gas emissions, and promote grain production.

Urease is a key catalytic enzyme involved in urea hydrolysis. Urease activity is closely related to temperature, water content, and nitrogen application rate ${ }^{[6-8]}$. The effect of individual environmental factors on urease activity has been revealed, but the interaction effect of the three mentioned factors is still unclear. Urea hydrolysis process is often described as a first-order kinetic model (Equation (1)) ${ }^{[9,10]}$. The hydrolysis rate constant $\left(K_{u}\right)$ is an important parameter in chemical reaction kinetics. In agriculture, the different soil temperatures, moisture contents, and nitrogen application rates can exert different impact degrees on urea hydrolysis ${ }^{[11-13]}$. Temporal and spatial variabilities of environmental conditions are found in soils. However, the value of $K_{u}$ at a fixed moisture content, temperature, and nitrogen concentration is often used in the simulation studies on nitrogen transformation and transportation ${ }^{[14-16]}$. This method reduces the accuracy of numerical simulations. The interaction effect of the three above-mentioned factors on $K_{u}$ should be further studied. Revealing the relationship between $K_{u}$ and environmental factors and establishing the modified model of $K_{u}$ can supplement the control mechanism of urea hydrolysis and improve the prediction accuracy of $K_{u}$.

$$
\frac{\partial U}{\partial t}=-K_{u} * U
$$

where, $U$ is the urea content $(\mathrm{mg} / \mathrm{kg})$ at time $t(\mathrm{~d}) ; K_{u}$ is the 
hydrolysis rate constant, $\mathrm{d}^{-1}$.

The speed of urea hydrolysis rate is closely related to temperature ${ }^{[12]}$. The relationship between $K_{u}$ and temperature can be described by the Arrhenius equation ${ }^{[7]}$. Arrhenius argued that the logarithmic value $\ln \left(K_{u}\right)$ of the hydrolysis rate constant $\left(K_{u}\right)$ is linearly related to the reciprocal $(1 / T)$ of the thermodynamic temperature $(T)$ (Equation (2) $)^{[7]}$. Equation (2) can also be simplified to another simple linear form (Equation (3) $)^{[17]}$. The value of $\ln \left(K_{u}\right)$ and $1 / T$ do not show a good linear relationship ${ }^{[18-20]}$. The reason is attributable to interference from other factors in the soil. Therefore, the Arrhenius model needs to be improved reasonably to contain additional critical environmental factors. Accordingly, the quantitative relationship between the factors and the rate constant can be expressed accurately.

$$
\begin{gathered}
\ln \left(K_{u}\right)=\ln (A)-\frac{E a}{R * T_{r}} \\
\ln \left(K_{u}\right)=a+\frac{b}{T_{r}}
\end{gathered}
$$

where, $A$ is the pre-exponential factor; $E_{a}$ is the activation energy, $\mathrm{kJ} / \mathrm{mol} ; R$ is the gas constant $(8.314 \mathrm{~J} /(\mathrm{mol} \cdot \mathrm{K})) ; a$ and $b$ are constants; $T_{r}$ is thermodynamic temperature, $\mathrm{K}$.

The effects of moisture content and nitrogen application rate on urea hydrolysis rate cannot be ignored ${ }^{[11-21]}$. The rate of urea hydrolysis in soil can decrease with the increase in water content, that is, a significant negative correlation exists between the two factors $^{[11]}$. A significant negative correlation also exists between the nitrogen application and hydrolysis rate ${ }^{[22]}$. This relationship is due to the non-competitive substrate inhibition of urease and the inactivation of urease ${ }^{[13,23]}$. However, traditional Arrhenius model and first-order kinetic model did not consider the effects of moisture content and nitrogen application rate on $K_{u}$. In conclusion, temperature, moisture content, and nitrogen application rate are the key influential factors of urea hydrolysis and should therefore be considered in establishing the hydrolysis parameter model.

Using the sample data of the laboratory culture experiments, this study aimed at revealing the coupling effect of temperature, moisture content, and nitrogen application rate on urease activity and hydrolysis parameters and establish a high-precision parametric model of $K_{u}$ for the simulation studies of nitrogen transformation and transportation.

\section{Materials and methods}

\subsection{Materials}

Soil was sampled from the Pomology Institute of the Shanxi Academy of Agricultural Sciences, and the samples were air dried and crushed to pass a 10-mesh sieve. Soil type was sandy loam, and $\mathrm{pH}$ was 8.84. The moisture content and field capacity were $21.63 \mathrm{~g} / \mathrm{kg}$ and $217.79 \mathrm{~g} / \mathrm{kg}$, respectively. The $\mathrm{NO}_{3}{ }^{-} \mathrm{N}$ and $\mathrm{NH}_{4}{ }^{+}-\mathrm{N}$ contents were $30.2 \mathrm{mg} / \mathrm{kg}$ and $25.7 \mathrm{mg} / \mathrm{kg}$, respectively. All chemicals used in the experiments were of analytical reagent grade and obtained from Sinopharm Chemical Reagent Beijing Co., Ltd. (Beijing, China).

\subsection{Treatment}

This study was mainly performed at the soil laboratory of Taiyuan University of Technology. The laboratory culture method was used to study the soil urea hydrolysis under different water contents, nitrogen application rates, and temperatures. Moisture content included three levels of $\theta_{60}, \theta_{80}$, and $\theta_{100}$, which corresponded to $60 \%, 80 \%$, and $100 \%$ of field capacities, respectively. Nitrogen application rate was divided into four levels of $F_{247}, F_{309}, F_{371}$, and $F_{433}$, which corresponded to $247 \mathrm{mg} / \mathrm{kg}$, $309 \mathrm{mg} / \mathrm{kg}, 371 \mathrm{mg} / \mathrm{kg}$, and $433 \mathrm{mg} / \mathrm{kg}$, respectively. Temperature was divided into four levels of $T_{15}, T_{20}, T_{25}$, and $T_{35}$, which referred to $15^{\circ} \mathrm{C}, 20^{\circ} \mathrm{C}, 25^{\circ} \mathrm{C}$, and $35^{\circ} \mathrm{C}$, respectively. This experiment included 48 treatments. All treatments were repeated three times. A total of $150 \mathrm{~g}$ (oven-dry basis) of soil was added to a series of $500 \mathrm{~mL}$ glass beakers. The beakers were treated with urea solution and deionized water to adjust the design level. The beakers were covered with a polyethylene film with several tiny holes. The beakers were incubated at different temperatures $\left(15^{\circ} \mathrm{C}, 20^{\circ} \mathrm{C}, 25^{\circ} \mathrm{C}\right.$, and $\left.35^{\circ} \mathrm{C}\right)$ for 9 days. The loss of soil water through evaporation was replenished daily with deionized water. The urea contents under different treatments were measured daily.

Soil urea hydrolysis rate is closely related to urease activity. Thus, a urease activity experiment should be conducted to reveal the mechanism of urea hydrolysis. In the urease activity experiment, the setting of various factors and levels was the same as those in the urea hydrolysis experiment. To prevent microbial proliferation, $30 \mathrm{~mL}$ of toluene was added to $150 \mathrm{~g}$ of soil sample and allowed to stand for $15 \mathrm{~min}$. Then, urea solution and the citrate buffer solution with $\mathrm{pH} 6.7$ were added to the soil to reach the design level of the soil urea concentration and water content. The beakers were incubated at different temperatures $\left(15^{\circ} \mathrm{C}, 20^{\circ} \mathrm{C}\right.$, $25^{\circ} \mathrm{C}$, and $35^{\circ} \mathrm{C}$ ) for $24 \mathrm{~h}$. In addition, a non-urea group was set as a blank control. Urease activity was expressed as milligrams of ammonium nitrogen released per kilogram of soil in $24 \mathrm{~h}$.

\subsection{Determination method}

Determination of urea content: $5 \mathrm{~g}$ (oven-dry basis) of soil and $50 \mathrm{~mL}$ of ultrapure water were added into the flasks, placed inside a mechanical shaker for $0.5 \mathrm{~h}$, and oscillated at a frequency of $50 \mathrm{~Hz}$. The soils and solution were separated by filtration with a filter membrane $(0.45 \mu \mathrm{m})$, and the urea content in the pellucid filtrate was determined through high-performance liquid chromatography (Thermo Fisher Scientific Corporation, America). The specific parameter setting was as follows: sample size of $30 \mu \mathrm{L}$, flow rate of $1 \mathrm{~mL} / \mathrm{min}$, mobile phase (95\% ultrapure $+5 \%$ acetonitrile), and chromatographic column $(\mathrm{C} 18,4.6 \mathrm{~mm} \times 150 \mathrm{~mm}$, $3 \mu \mathrm{m}$ ) with a chromatographic column temperature of $30^{\circ} \mathrm{C}$ and wavelength of $190 \mathrm{~nm}$.

Determination of $\mathrm{NH}_{4}{ }^{+}-\mathrm{N}$ contents: 5 grams (oven-dry basis) of soil and $100 \mathrm{~mL}$ of $\mathrm{KCl}$ solution $(1 \mathrm{~mol} / \mathrm{L})$ were added into flasks and then placed inside a mechanical shaker and oscillated at a frequency of $50 \mathrm{~Hz}$ for $1 \mathrm{~h}$. The soils and solution were separated by filtration with a filter membrane $(0.22 \mu \mathrm{m})$, and the $\mathrm{NH}_{4}{ }^{+}-\mathrm{N}$ contents in the pellucid filtrate were determined with a continuous flow analyzer (AA3, Bran Luebbe, Germany).

\subsection{Data processing}

A first-order model (Equation (1)) based on kinetic theory was used to describe the dynamic process of urea hydrolysis. $E_{a}$ can be calculated by Equation (2). A regression analysis was conducted using the 1stopt 7.0 software to calculate the parameters of the models from the experimental data. The three-way ANOVA was performed with IBM SPSS Statistics 19 software, and the figures were plotted with Origin 9.1 software. The simulation effects of the models were evaluated using the mean absolute percentage error (MAPE) and the determination coefficient $\left(R^{2}\right)$, which were calculated as follows:

$$
M A P E=\frac{1}{n} \sum_{i=1}^{n}\left|\frac{C_{i}-C_{i}^{\prime}}{C_{i}}\right| \times 100 \%
$$




$$
R^{2}=\sum_{i=1}^{n}\left(C_{i}^{\prime}-\bar{C}\right)^{2} / \sum_{i=1}^{n}\left(C_{i}-\bar{C}\right)^{2}
$$

where, $C_{i}, C_{i}^{\prime}$, and $\bar{C}$ represent the measured, calculated, and average value of $K_{u}\left(\mathrm{~d}^{-1}\right)$, respectively; $n$ is the number of evaluated data points.

\section{Results}

\subsection{Urease activity and hydrolysis rate constant under different treatments}

Urease is a catalyst for urea hydrolysis, and its activity is indicative of the urea hydrolysis rate. Figure 1 shows the urease activity $\left(V_{0}\right)$ under the coupling condition of moisture content, nitrogen application rate, and temperature. When temperature increased from $T_{15}$ to $T_{35}$, and nitrogen application rate increased from $F_{247}$ to $F_{433}$, the soil urease activity showed an increasing trend on power function. The soil urease activity increased by $0.15-0.62$ times and 0.19-0.40 times in this process, indicating that the increased temperature and nitrogen application rate promoted urease activity. The urease activity treated with $\theta_{100}$ was 0.77-0.98 times of that of $\theta_{60}$, which indicated that soil urease activity was inhibited when the soil water content increased. The three-way ANOVA of $V_{0}$ was performed to reveal the coupling effect of the three factors, and the results are shown in Table 1. The table shows that the effect of single factor and two-factor coupling on urease activity was extremely significant $(p<0.01)$, whereas the effect of the three-factor coupling on urease activity was trivial. The effects of three factors and their interactions on urease activity were in the descending order: $T, F, \theta, T^{*} F, T^{*} \theta, F^{*} \theta$, $T^{*} \theta * F$.

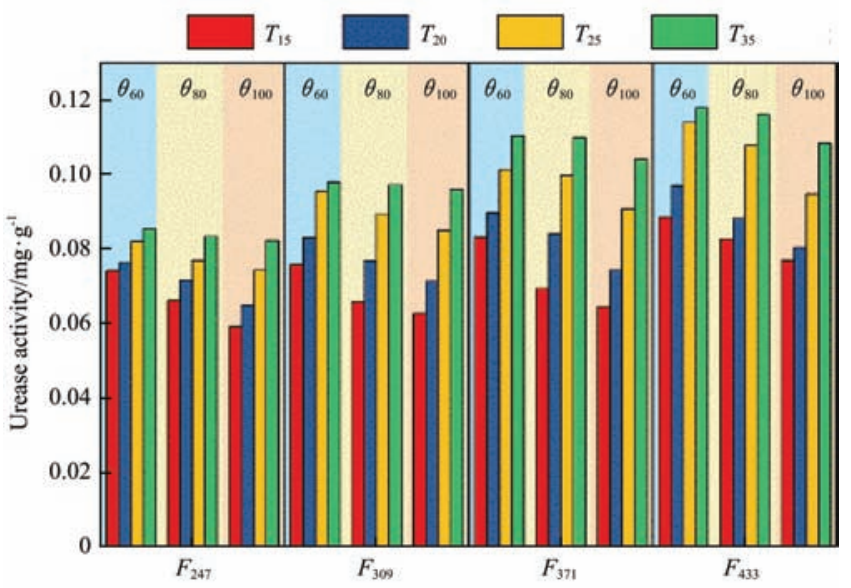

Figure 1 Urease activity under the coupled effect of water-nitrogen-temperature

Table 1 Three-way ANOVA of urease activity and urea hydrolysis rate constant under the coupled effect of water-temperature-nitrogen

\begin{tabular}{cccccccc}
\hline & \multicolumn{3}{c}{ Urease activity $\left(V_{0}\right)$} & & \multicolumn{2}{c}{ Hydrolysis rate constant $\left(K_{u}\right)$} \\
\cline { 2 - 4 } \cline { 6 - 8 } Source & $\begin{array}{c}\text { Sum of } \\
\text { squares }\end{array}$ & F-value & P-Value & & $\begin{array}{c}\text { Sum of } \\
\text { squares }\end{array}$ & F-value & P-Value \\
\hline$T$ & 0.017 & 378.082 & $<0.001$ & 74769.514 & 6697.022 & $<0.001$ \\
$F$ & 0.010 & 222.873 & $<0.001$ & 30354.741 & 1284.150 & $<0.001$ \\
$\theta$ & 0.003 & 85.860 & $<0.001$ & 7394.418 & 112.203 & $<0.001$ \\
$T^{*} F$ & $1.313 \mathrm{E}-3$ & 9.483 & $<0.001$ & 1480.021 & 30.944 & $<0.001$ \\
$T^{*} \theta$ & $6.547 \mathrm{E}-4$ & 7.095 & $<0.001$ & 255.124 & 8.001 & $<0.001$ \\
$F^{*} \theta$ & $6.487 \mathrm{E}-4$ & 7.112 & $<0.001$ & 112.198 & 4.692 & $<0.001$ \\
$T^{*} F^{*} \theta$ & $4.075 \mathrm{E}-5$ & 1.472 & 0.117 & 101.005 & 4.547 & $<0.001$ \\
\hline
\end{tabular}

Figure 2 shows the hydrolysis rate constant $\left(K_{u}\right)$ under the coupling effect of moisture content, nitrogen application rate, and temperature. When the soil temperature increased from $T_{15}$ to $T_{35}$, the value of $K_{u}$ increased by 1.96-2.66 times, indicating that temperature had a significant effect on $K_{u}$. At the same level of nitrogen application rate and moisture content, the increase in temperature increased the urease activity (Figure 1), which in turn promoted the urea hydrolysis rate $(\partial U / \partial t)$ and decreased the residual substrate concentration $(U) . \quad K_{u}$ must increase with the increase in temperature according to Equation (1).

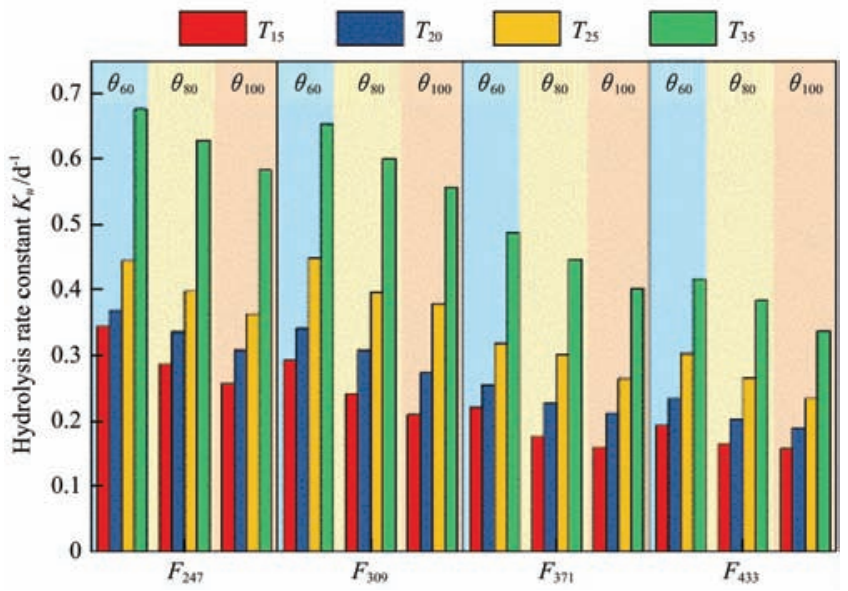

Figure 2 Urea hydrolysis rate constant $\left(K_{u}\right)$ under the coupled effect of water-nitrogen-temperature

When soil moisture content increased from $\theta_{60}$ to $\theta_{100}, K_{u}$ decreased by $13.9 \%-28.7 \%$. At the same level of nitrogen application rate and temperature, the increase in moisture content inhibited the urease activity (Figure 1), which in turn decreased the urea hydrolysis rate $(\partial U / \partial t)$ and increased the residual substrate concentration $(U) . \quad K_{u}$ must decrease with the increase in moisture content according to Equation (1).

In analyzing the response of nitrogen application rate to $K_{u}$, the increase in nitrogen application rate and $V_{0}$ could not account for the relative size of the residual substrate concentration because of the different initial nitrogen application rates. At this point, the dynamic process of the substrate concentration should be analyzed. During the entire hydrolysis process, when the nitrogen application rate increased from $F_{247}$ to $F_{433}$, the residual substrate concentration $(U)$ increased by $1.75-16.83$ times (Figure 1 ) whereas $K_{u}$ decreased by $0.56-0.68$ times (Figure 2); the product of the two must be greater than 1 . Therefore, high nitrogen application rate means high urease activity $\left(V_{0}\right)$, high concentration $(U)$, and low constant $\left(K_{u}\right)$. The three-way ANOVA of $K_{u}$ was performed to reveal the coupling effect of the three factors, and the results are shown in Table 1. Table 1 shows that the effects of three factors and their interaction on $K_{u}$ were extremely significant $(p<0.01)$. The effects of three factors and their interaction on $K_{u}$ were in the descending order: $T, F, \theta, T^{*} F, T^{*} \theta, F^{*} \theta, T^{*} \theta^{*} F$. Therefore, the effects of individual factors and multi-factor interaction on $K_{u}$ should be considered in establishing a quantitative model.

\subsection{Activation energy $\left(E_{a}\right)$ of urea hydrolysis under different treatments}

$E_{a}$ is an important thermodynamic indicator that characterizes the difficulty in chemical reactions. This indicator reflects the energy barriers that need to be overcome when urea hydrolysis occurs. Figure 3 shows $E_{a}$ calculated by the Arrhenius equation under different treatments. $E_{a}$ increased exponentially with the 
increase in nitrogen application rate and linearly with moisture content. $E_{a}$ had a stronger response to nitrogen application rate than to moisture content. When the moisture content and nitrogen application rate increased, the energy barrier $\left(E_{a}\right)$ that must be overcame also increased. Consequently, $K_{u}$ decreased. The effects of moisture content, nitrogen application rate, and their interaction on $E_{a}$ were in the descending order: $F, \theta, F^{*} \theta$. The effects of two factors and their interaction on $E_{a}$ were not significant.

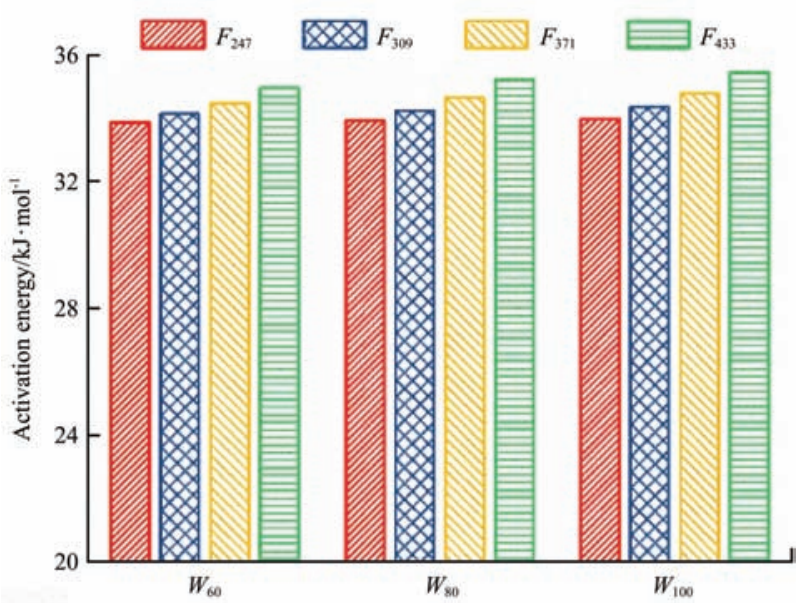

Figure 3 Activation energy $\left(E_{a}\right)$ under the coupled effect of water-nitrogen

\subsection{Establishment of the coupling model of $K_{u}$}

From the experiment results, the relationship between $K_{u}$ and moisture content, nitrogen application rate, and temperature can be described by Equations (6)-(8), respectively. The average values of determination coefficient of the three models were $0.977,0.963$, and 0.994 , respectively; this finding showed a satisfactory fitting accuracy. Considering the existence of the interaction between the three factors, Equations (6)-(8) were multiplied to obtain a quantitative model $K_{u-1}(\theta, T, F)$ of $K_{u}$ under the coupling condition of temperature, moisture content, and nitrogen application rate. The $K_{u-1}(\theta, T, F)$ model was shown as Equation (9).

$$
\begin{aligned}
& K_{u}(\theta)=K_{\mathrm{u} \theta} * \exp (a *|b-\theta|) \\
& K_{u}(T)=K_{u T} * \exp (a *|b-T|) \\
& K_{u}(F)=K_{u F} * \exp (a *|b-F|)
\end{aligned}
$$

$K_{u-1}(\theta, F, T)=K_{u \theta F T} * \exp (a *|b-\theta|+c *|d-F|+e *|f-T|)(9)$ where, $K_{u \theta}, K_{u T}$, and $K_{u F}$ are the values of $K_{u}$ under standard moisture content $\left(\theta_{100}\right)$, standard temperature $\left(T_{15}\right)$, and standard nitrogen application rate $\left(F_{433}\right)$, respectively; $\theta, T$, and $F$ are the moisture content $(\mathrm{g} / \mathrm{kg})$, temperature $\left({ }^{\circ} \mathrm{C}\right)$, and nitrogen application rate $(\mathrm{mg} / \mathrm{kg})$, respectively; $K_{u \theta T F}$ is the value of $K_{u}$ under the standard coupling condition of the three factors $\left(\theta_{100}, T_{15}, F_{433}\right) ; a$, $b, c, d, e$, and $f$ are constants.

Another three-factor coupling model $K_{u-2}(\theta, T, F)$ of $K_{u}$ was established in this study. According to Arrhenius' theory, the relationship between $\ln \left(K_{u}\right)$ and $1 / T$ can be described by Equation $(3)^{[17]}$. The determination coefficient $\left(R^{2}\right)$ of the linear model between $\ln \left(K_{u}\right)$ and 1/T was 0.93-0.98. Therefore, the Arrhenius equation $K_{u-3}(T)$ can be applied to soil urea hydrolysis. After a reasonable improvement, the accuracy of $K_{u-3}(T)$ model may be further improved. The classical Arrhenius model $K_{u-3}(T)$ only represents the relationship between temperature and $K_{u}$. However, the effects of moisture content and nitrogen application rate on $K_{u}$ were very significant $(p<0.01)$. Therefore, the influence of moisture content and nitrogen application rate should be considered in improving the $K_{u-3}(T)$ model. Given the significant interaction between the three factors, Equations (3), (6) and (8) were multiplied to obtain another coupling model $K_{u-2}(\theta, T, F)$ of $K_{u}$ based on Arrhenius' model. The $K_{u-2}(\theta, T, F)$ model was shown as Equation (10).

$$
K_{u-2}(\theta, T, F)=a * \exp (b *|c-\theta|+d *|e-F|+f / T)
$$

\subsection{Calibration and verification of the coupling model of $\boldsymbol{K}_{\boldsymbol{u}}$}

The parameters of $K_{u-1}(\theta, T, F)$ and $K_{u-2}(\theta, T, F)$ were determined by $80 \%$ of the total samples. Table 2 shows the specific parameter, and Figure 4 shows the linear consistency and relative error between the observed and calculated values of the calibration samples. The linear consistency of $K_{u-1}(\theta, T, F)$ model was the best, that of the $K_{u-2}(\theta, T, F)$ model followed, and that of the $K_{u-3}(T)$ model was the worst (Figure 4a). The MAPE values of the $K_{u-1}(\theta, T, F)$ model, $K_{u-2}(\theta, T, F)$, and $K_{u-3}(T)$ were $2.89 \%, 3.67 \%$, and $5.36 \%$, respectively. In conclusion, the calibration effects of the three models were in the descending order: $K_{u-1}(\theta, T, F), K_{u-2}(\theta, T, F), K_{u-3}(T) . \quad K_{u}$ was predicted using the three models which had been calibrated and the verification samples $(20 \%$ of the total samples). The prediction results in Figure 5 show that the consistency between the observed and predicted values calculated by the $K_{u-1}(\theta, T, F)$ model was better than that of the two other models. For the verification samples, the MAPE values of $K_{u-1}(\theta, T, F), K_{u-2}(\theta, T, F)$, and $K_{u-3}(T)$ models were $3.14 \%, 4.60 \%$, and $6.75 \%$, respectively. The $K_{u-1}(\theta, T, F)$ model described the relationship between $K_{u}$ and the three factors better than the two other models did. Thus, the $K_{u-1}(\theta, T, F)$ model was recommended for the quantitative studies.

Table 2 Fitting parameters of $K_{u-1}(\theta, T, F)$ model and $K_{u-2}(\theta, F, T)$ model

\begin{tabular}{cccccccc}
\hline Model & $K_{u \theta T F}$ & $a$ & $b$ & $c$ & $d$ & $e$ & $f$ \\
\hline$K_{u-1}(\theta, T, F)$ & 0.157 & $2.251 \mathrm{E}-3$ & 834.033 & $2.885 \mathrm{E}-3$ & 422.949 & -0.041 & 51.016 \\
$K_{u-2}(\theta, F, T)$ & & 0.659 & $2.348 \mathrm{E}-3$ & 192.898 & $2.864 \mathrm{E}-3$ & 423.073 & -24.507 \\
\hline
\end{tabular}

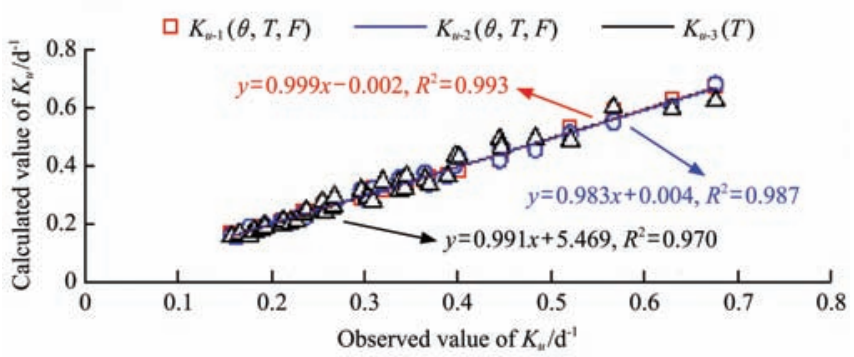

a. Observed and calculated values of $K_{u}$

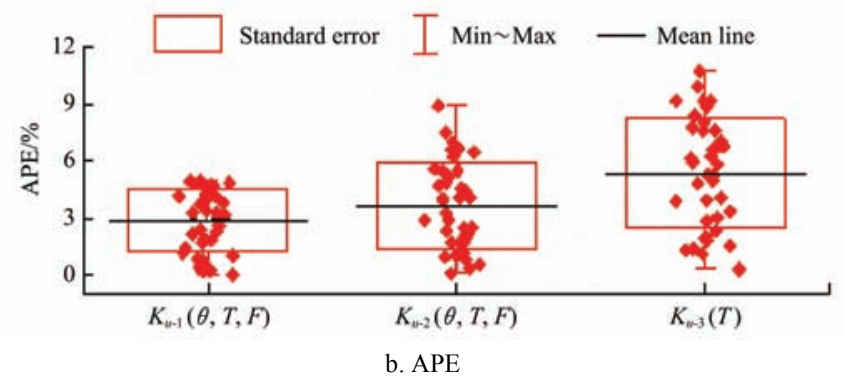

Figure 4 Linear relationship and absolute percentage error (APE) between observed and calculated value obtained from the three models 

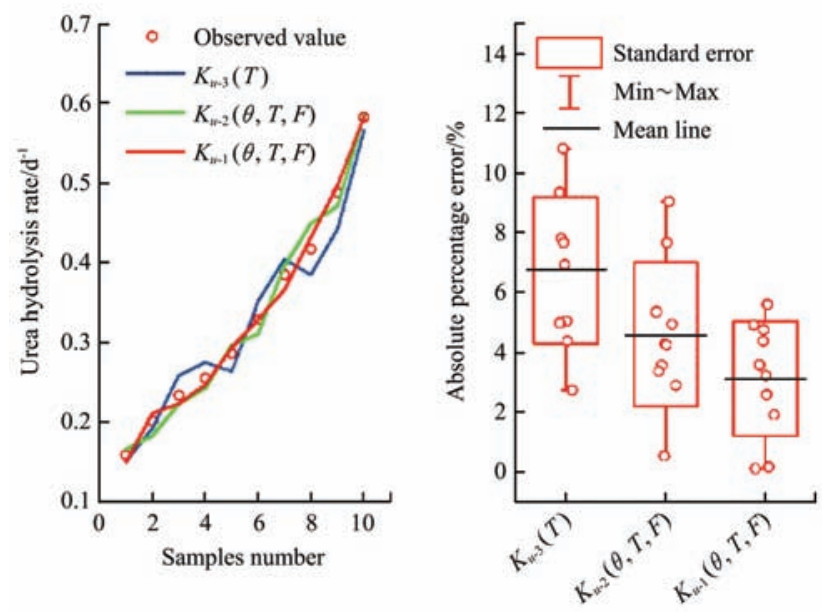

Figure 5 Comparison between observed and calculated value obtained from the three models

\section{Discussion}

\subsection{Determination method of urea content}

In many studies on urea hydrolysis characteristics, the urea content is often determined by the diacetyl monooxime method $^{[24-26]}$. This method is complicated and time consuming. The soil extraction and the various chemical reagents need to be mixed, and the reaction must be completed first prior to the determination. The sensitivity of the method is $0.04 \mathrm{mg} / \mathrm{L}^{[26]}$. The method of high performance liquid chromatography, which belongs to the physical separation method, was used in this study. This method can directly determine the urea content of soil extraction and avoid the systematic error caused by the chemical treatment process unlike previous reports. The sensitivity of this method is $0.006 \mathrm{mg} / \mathrm{L}$. Compared with those of traditional methods, the results of this study would be more accurate and reliable.

\subsection{Urease activity and hydrolysis rate constant under different treatments}

When moisture content increased from $50 \%$ to $100 \%$ of field capacities, the urease activity increased from $0.22 \mathrm{mg} / \mathrm{g}$ to $0.28 \mathrm{mg} / \mathrm{g}$ and the percentage of increase was $26.2 \%{ }^{[6]}$. This previous finding is contrary to the results of this study. When moisture content increased from $60 \%$ to $100 \%$ of field capacities, the urease activity decreased by $2.1 \%$ to $22.5 \%$. This difference might be due to different soil particle gradations. The previous proportions of soil sand, granule, and clay are $66.5 \%, 17.5 \%$, and $16.0 \%$. By contrast, the present results were $34.6 \%, 51.5 \%$ and $13.9 \%$. Thus, the texture of the two soil samples differed. Given the strong viscosity of soil, the effects of increased moisture content on soil air permeability was larger than that in the previous report. In the current study, the increase in moisture content indicated worse air permeability, low urease activity, and slow hydrolysis rate.

This study showed a positive correlation between temperature and urease activity, which is similar to that of MOYO et al. ${ }^{[7]}$ However, the response of temperature to urease activity in the present study differed from that in the previous study. MOYO ${ }^{[7]}$ showed that the urease activity of Kahola and Smoltm soils increases two and three times, respectively, when temperature increases from $15^{\circ} \mathrm{C}$ to $35^{\circ} \mathrm{C}$. However, the current results were between 0.15 and 0.62 times. The difference of the results was due to the different nitrogen application rates in the two studies.
In this study, the nitrogen application rate was from $247 \mathrm{mg} / \mathrm{kg}$ to $433 \mathrm{mg} / \mathrm{kg}$, and the urease activity did not reach saturation. On the contrary, in MOYO's study, the nitrogen application rate is $1000 \mathrm{mg} / \mathrm{kg}$, and the urease activity is saturated ${ }^{[7]}$. The response intensity of the urease activity to temperature might differ under different nitrogen fertilizer concentrations. Urease activity depends mainly on the soil temperature and moisture content but not on the soil nitrogen concentration ${ }^{[8]}$. In this study, not only the temperature and moisture content had a significant effect on urease activity but also the nitrogen application rate. This finding slightly differed from those of previous research. Sardans's research was carried out in the field, and the results may be disturbed by other factors ${ }^{[8]}$. Meanwhile, this study was carried out under a factor-controllable laboratory experiment, and the results could be reliable. When urea concentration increased, the urea molecules adsorbed in the urease activity site and the interface between the two increased. Therefore, the hydrolysis rate was promoted.

The effect of single factor on urease activity is currently being explored $^{[6-8,12]}$, and the studies reveal the effect of single factors on urease activity but ignore the effect interaction between the factors. In this research work, a supplementary study was conducted to reveal the interaction effect of temperature, moisture content, and nitrogen application rate on urea hydrolysis. The effects of the three factors and their interaction on $V_{u}$ were in the descending order: $T, F, \theta, T^{*} F, T^{*} \theta, F^{*} \theta, T^{*} \theta^{*} F$.

\subsection{Kinetics of urea hydrolysis under different treatments}

Hydrolysis rate $(\partial U / \partial t)$ increases first and then decreases with the increase in urea concentration ${ }^{[23]}$, which is different from this study's result. This study showed that hydrolysis rate $(\partial U / \partial t)$ enhanced with the increase in urea concentration. In Singh's report ${ }^{[23]}$, the moisture content was $50 \%$ and nitrogen application rate was less than $200 \mathrm{mg} / \mathrm{kg}$. However, the moisture content was between $19.2 \%$ (60\% of field capacities) and $32 \%$ $(100 \%$ of field capacities) and nitrogen application rate was between $247 \mathrm{mg} / \mathrm{kg}$ and $433 \mathrm{mg} / \mathrm{kg}$ in this study. The result difference between the two studies might be due to the different urease activity to nitrogen application rate under different moisture contents and nitrogen application rates. In Singh's study ${ }^{[23]}$, the soil water content is relatively high, soil permeability is poor, urease is strongly inhibited, and its activity level is low. At this point, the increase in nitrogen application rate may have toxic effects on urease activity, thereby reducing the hydrolysis rate. In the current study, the moisture content was acceptable, and the increase in nitrogen application rate might increase the urease activity $\left(V_{0}\right)$, thereby promoting the hydrolysis rate $(\partial U / \partial t)$.

The current soil temperature was positively correlated with hydrolysis rate $(\partial U / \partial t)$, which was similar to the previous study ${ }^{[12,27,28]}$. This relationship was mainly caused by three reasons. First, when the temperature increased from $15^{\circ} \mathrm{C}$ to $35^{\circ} \mathrm{C}$, the urease activity increased by $0.15-0.62$ times (Figure 1 ), thereby accelerating urea hydrolysis. Second, when the temperature increased from $15^{\circ} \mathrm{C}$ to $35^{\circ} \mathrm{C}$, the diffusion amount of ammonium nitrogen from the liquid phase to the gas phase increased by 3.2 to 17.9 times (Figure 2), thereby resulting in the right moving of the chemical reaction balance (Equation (11)) and the increase in hydrolysis rate ${ }^{[29]}$. Third, the increase in temperature increased the percentage of activated molecules and the probability of effective collision between molecules; these conditions promoted the hydrolysis reaction ${ }^{[30]}$. 


$$
\mathrm{CO}\left(\mathrm{NH}_{2}\right)_{2}+\mathrm{H}_{2} \mathrm{O} \rightarrow 2 \mathrm{NH}_{3}+\mathrm{CO}_{2}
$$

The effects of single factors on kinetics of urea hydrolysis are currently being examined, thereby revealing the relationship between the factors and kinetic parameters ${ }^{[12,23,27,28]}$. However, the interaction effect of the three factors on the kinetics of urea hydrolysis should be further explored. In this study, the effects of three factors and their interaction on $K_{u}$ were in the descending order: $T, F, \theta, T^{*} F, T^{*} \theta, F^{*} \theta, T^{*} \theta^{*} F$. Moreover, the effect of three individual factors and their interaction on $K_{u}$ was extremely significant $(p<0.01)$. These findings supplemented the mechanism of urea hydrolysis.

The established $K_{u-1}(\theta, T, F)$ and $K_{u-2}(\theta, T, F)$ models showed more general applicability and better prediction accuracy than the traditional $K_{u-3}(T)$ model. First, when the traditional $K_{u-3}(T)$ model was adopted to quantify the relationship between $K_{u}$ and the three factors (temperature, moisture content, and nitrogen application rate), 48 kinds of models had to be established to satisfy the different treatments. When the $K_{u-1}(\theta, T, F)$ and $K_{u-2}(\theta, T, F)$ models were adopted, one model could quantify the relationship between $K_{u}$ and the three factors with all treatments. Second, the accuracies of the three models were in the descending order: $K_{u-1}(\theta$, $T, F), K_{u-2}(\theta, T, F), K_{u-3}(T)$. The results improved the prediction accuracy of $K_{u}$. Among them, the $K_{u-1}(\theta, T, F)$ model was advised to be used in the quantitative study on urea because of its low MAPE value. On the one hand, the $K_{u-1}(\theta, T, F)$ model brought into Equation (1) could achieve the dynamic prediction of urea hydrolysis under the coupling effect of moisture content, temperature, and nitrogen application rate, thus improving the kinetic mechanism of urea hydrolysis. On the other hand, the $K_{u-1}(\theta, T, F)$ model could be used as a source and sink term of the quantitative model of nitrogen transformation and transportation. Given that the $K_{u-1}(\theta, T, F)$ model considered the effect of environmental factors on hydrolysis, the accuracy of quantitative model of nitrogen transformation and transportation could be improved.

The difference in $E_{a}$ between different reports is obvious. The value of $E_{a}$ is $96.2 \mathrm{~kJ} / \mathrm{mol}$ in Rachinskii's report ${ }^{[31]}$ and $41.0 \mathrm{~kJ} / \mathrm{mol}$ in Gould's report ${ }^{[32]}$, respectively. In this study, the average value of $E_{a}$ was $34.5 \mathrm{~kJ} / \mathrm{mol}$. This result was much smaller than the value reported by Rachinskii ${ }^{[31]}$. The observed variations could be due to differences in the techniques used in the two studies. The urea content is determined by the diacetyl monooxime method in Rachinskii's report ${ }^{[31]}$. By contrast, the method of high performance liquid chromatography was used in this study. Various assay techniques can induce conditions that influence differently the energy requirements for the formation of enzyme-substrate complex and thus the activation energy. The present results slightly differed from the findings reported by Gould $^{[32]}$. However, the current urea hydrolysis was consistent with the first-order kinetics in Gould's study ${ }^{[32]}$. The control mechanism of the two studies was same. On the other hand, the experimental conditions differed between the two studies. The previous moisture content and nitrogen application are fixed at $24 \%$ and $200 \mathrm{mg} / \mathrm{kg}$, respectively, unlike those in the present study. Previous studies only consider the temperature effect. By contrast, the current study not only considered the effects of moisture content, temperature, and nitrogen application rate but also the coupling effect among the three factors. The present results might be more accurate and reliable than the findings of the previous studies.

\section{Conclusions}

(1) The effects of moisture content, temperature, nitrogen application rate, and their interaction on $V_{0}$ and $K_{u}$ were in the descending order: $T, F, T^{*} F, \theta, T^{*} \theta, F^{*} \theta, T^{*} \theta^{*} F$. The effects of single factor and two-factor coupling on $V_{0}$ were extremely significant $(p<0.01)$, whereas the effect of the three-factor coupling on $V_{0}$ was insignificant. The effects of three factors and their interaction on $K_{u}$ were extremely significant $(p<0.01)$. The results represent an important stepping stone toward good understanding of the key mechanism of urea hydrolysis.

(2) The effects of moisture content, nitrogen application rate, and their interaction on $E_{a}$ were in the descending order: $F, \theta, F^{*} \theta$. The effects of two factors and their interaction on $E_{a}$ were not significant. These findings supplemented the mechanism of urea hydrolysis.

(3) The MAPE values of the established $K_{u-1}(\theta, T, F)$ and $K_{u-2}(\theta, T, F)$ models were $3.14 \%$ and $4.60 \%$, respectively; the MAPE of the traditional Arrhenius model $K_{u-3}(T)$ was $6.75 \%$. The accuracy of the proposed three-factor interaction model was superior to that of the traditional single factor model. The development of these two models represents an important stepping stone toward good understanding of the relationship between $K_{u}$ and environmental factors. Therefore, this study provides a valuable way to predict urea hydrolysis rate.

\section{Acknowledgements}

This study is supported by the National Natural Science Foundation of China (No. 51579168 and No. 51249002), the Natural Science Foundation of Shanxi Province of China (No. 201601D011053), the Graduate Education Innovation Program of Shanxi Province of China (No. 2016BY064), and the Scientific and Technological Project of Shanxi Province of China (No. 20140311016-6).

\section{[References]}

[1] Wang L L, Li W Z, Wang Z J, Wang Z W, Sui C, Li Y. Effects of digestate application depth on soil nitrogen volatilization and vertical distribution. Int J Agric \& Biol Eng, 2016; 9(5): 101-107.

[2] Zhao Z P, Duan M, Yan S, Liu Z F, Wang Q, Fu J, et al. Effects of different fertilizations on fruit quality, yield and soil fertility in field-grown kiwifruit orchard. Int J Agric \& Biol Eng, 2017; 10: 162-171.

[3] Zhao Z P, Yan S, Liu F, Ji P H, Wang X Y, Tong Y A. Effects of chemical fertilizer combined with organic manure on Fuji apple quality, yield and soil fertility in apple orchard on the Loess Plateau of China. Int J Agric \& Biol Eng, 2014; 7: 45-55.

[4] Wang C F, Shao X H, Xu H L, Chang T T, Wang W N. Effects of compound microbial inoculant treated wastewater irrigation on soil nutrients and enzyme activities. Int J Agric \& Biol Eng, 2016; 9: 100-108.

[5] Yang X, Wu L, Wu S, Chen J. Nitrogen release characteristic of polymer coated urea in paddy soil and its relationship with cumulative temperature. Transactions of the CSAE, 2016; 32: 199-204. (in Chinese)

[6] Antil D R S, Mahata M K, Narwal R P. Effect of substrate concentration, soil moisture, and organic materials on urease activity of soil contaminated with lead. Archives of Agronomy \& Soil Science, 2006; 52: 61-68.

[7] Moyo C C, Kissel D E, Cabrera M L. Temperature effects on soil urease activity. Soil Biology \& Biochemistry, 1989; 21: 935-938.

[8] Sardans J, PeñUelas J, Estiarte M. Changes in soil enzymes related to C and $\mathrm{N}$ cycle and in soil $\mathrm{C}$ and $\mathrm{N}$ content under prolonged warming and drought in a Mediterranean shrubland. Applied Soil Ecology, 2008; 39: 223-235.

[9] Liang X Q, Yuan YJ, He M M, Hua L, Liang L, Tian G M. Modeling the fate of fertilizer $\mathrm{N}$ in paddy rice systems receiving manure and urea. Geoderma, 2014; 228-229: 54-61.

[10] Rodríguez S B, Alonso-Gaite A, Álvarez-Benedí J. Characterization of 
nitrogen transformations, sorption and volatilization processes in urea fertilized soils. Vadose zone journal, 2005; 4: 329-336.

[11] Chauhan H S, Mishra B. Ammonia volatilization from a flooded rice field fertilized with amended urea materials. Fertilizer research, 1989; 19: $57-63$.

[12] Lal R, Kissel D, Cabrera M, Schwab A. Kinetics of urea hydrolysis in wheat residue. Soil Biology and Biochemistry, 1993; 25: 1033-1036.

[13] Sankhayan S D, Shukla U C. Rates of urea hydrolysis in five soils of India. Geoderma, 1976; 16: 171-178.

[14] Chowdary V M, Rao N H, Sarma P B S. A coupled soil water and nitrogen balance model for flooded rice fields in India. Agriculture, Ecosystems \& Environment, 2004; 103: 425-441.

[15] Liang X Q, Chen Y X, Li H, Tian G M, Ni W Z, He M M, et al. Modeling transport and fate of nitrogen from urea applied to a near-trench paddy field. Environmental Pollution, 2007; 150: 313-320.

[16] Liang X Q, Li Y J, He M M, Hua L, Liang L, Tian G M. Modeling the fate of fertilizer $\mathrm{N}$ in paddy rice systems receiving manure and urea. Geoderma, 2014; 228: 54-61.

[17] Todd R W, Cole N A, Waldrip H M, Aiken R M. Arrhenius equation for modeling feedyard ammonia emissions using temperature and diet crude protein. J Environ Qual, 2013; 42: 666-671.

[18] Dessureault-Rompré J, Zebarth B J, Georgallas A, Burton D L, Grant C A, Drury C F. Temperature dependence of soil nitrogen mineralization rate: Comparison of mathematical models, reference temperatures and origin of the soils. Geoderma, 2010; 157: 97-108.

[19] Frøseth R B, Bleken M A. Effect of low temperature and soil type on the decomposition rate of soil organic carbon and clover leaves, and related priming effect. Soil Biology and Biochemistry, 2015; 80: 156-166.

[20] Vourlitis G L, DeFotis C, Kristan W. Effects of soil water content, temperature and experimental nitrogen deposition on nitric oxide (NO) efflux from semiarid shrubland soil. Journal of Arid Environments, 2015; 117: $67-74$.
[21] Cabrera M L, Kissel D E, Bock B R. Urea hydrolysis in soil: Effects of urea concentration and soil pH. Soil Biology \& Biochemistry, 1991; 23: 1121-1124.

[22] Yadav D, Kumar V, Singh M, Relan P. Effect of temperature and moisture on kinetics of urea hydrolysis and nitrification. Soil Research, 1987; 25: 185-191

[23] Singh R, Nye P H. The effect of soil $\mathrm{pH}$ and high urea concentrations on urease activity in soil. European Journal of Soil Science, 1985; 35: 519-527.

[24] Dalal R. Urease activity in some Trinidad soils. Soil Biology and Biochemistry, 1975; 7: 5-8.

[25] Gou W, Zheng P F, Tian L, Gao M, Zhang L X, Akram N A, et al Exogenous application of urea and a urease inhibitor improves drought stress tolerance in maize (Zea mays L.). Journal of Plant Research, 2017; 130: 599-609.

[26] Sudkolai S T, Nourbakhsh F. Urease activity as an index for assessing the maturity of cow manure and wheat residue vermicomposts. Waste Management, 2017; 64: 63-66.

[27] Burns R G. Soil enzymes. London: Academic Press, 1978.

[28] Suter H C, Pengthamkeerati P, Walker C, Chen D. Influence of temperature and soil type on inhibition of urea hydrolysis by $\mathrm{N}$-(n-butyl) thiophosphoric triamide in wheat and pasture soils in south-eastern Australia. Soil Research, 2011; 49: 315-319.

[29] Pauling L. General chemistry. Massachusetts: Courier Corporation, 2014.

[30] Hartley I P, Ineson P. Substrate quality and the temperature sensitivity of soil organic matter decomposition. Soil Biology and Biochemistry, 2008; 40: $1567-1574$.

[31] Rachinskii V, Pelttser A. Effect of temperature on rate of decomposition of urea in soil. Agrokhimiya, 1967; 10: 75-77.

[32] Gould W, Cook F, Webster G. Factors affecting urea hydrolysis in several Alberta soils. Plant and Soil, 1973; 38: 393-401. 